\section{How does the brain do it?}

\author{
Stuart Sutherland
}

Visual Cognition. Edited by Steven Pinker. MIT Press: 1986. Pp.270. Pbk $\$ 17.50, £ 17.50$.

MANY years ago, I put forward the following problem. A cross and a circle, horizontally aligned, are flashed onto the retina; regardless of the shapes' absolute positions, the subject has to determine which is on the left. As visual tasks go, it could hardly be simpler: the problem is that we have no idea how the brain does it. A scan from left to right (or vice versa) seems improbable. If, on the other hand, the coordinates of each shape are carried forward, how is this done and how is the comparison between the coordinates made in neural terms?

In the most original and important article in Visual Cognition, Shimon Ullman points to a series of similar problems. He asks what mechanism could trace out a closed curve to decide that it is closed and to determine which points lie inside and which outside it. The problem is a more precise version of the figure-ground effect, so beloved by the Gestaltists. Although the question can be stated more rigorously than in their day, we are no nearer a solution, at least in terms of brain mechanisms, for there is no difficulty in writing computer programs to carry out this task.

At the other extreme of vision are complicated problems which still defy computers, but which are solved by the brain by means that remain obscure. Perhaps the most difficult is that of visual recognition. Steven Pinker gives a judicious review of the subject, noting the many difficulties that beset current theories. We have of course moved on: nowadays Gestalt theorizing looks hopelessly vague, and the idea that recognition could be achieved by templates or by feature matching, which was being put forward in all seriousness only 20 years ago, now looks extremely naive. Some of the more recent ideas will probably survive, for example that, for the purposes of recognition, the brain builds an abstract and hierarchical structural description of an object. However, the exact nature of such descriptions, the methods by which they are derived from the retinal image, and how or indeed whether the same description is formed of an object regardless of viewpoint remain entirely open questions.

Half of the papers collected by Pinker are devoted to visual imagery and the ways in which people manipulate it, a subject on which there has recently been much research. There are a limited num- ber of transformations that can be applied to the image of an object - it can, for example, be made to change size, to rotate or to change position. In all such changes, the imaged object passes through all intermediate points. One cannot suddenly replace an image of an upright letter with that of the same letter tilted through $90^{\circ}$. Steven Kosslyn has constructed an ingenious computer model which incorporates the processes required to manipulate imagery in the way people do. He describes his model and the results of submitting it to a series of tests, in which he uses consistent differences in people's ability to perform the different operations to demonstrate that they really are used by the brain.

Such questions as whether the tail of a horse ends above or below its knees can only be answered by using imagery. But if for the purpose of recognition we construct structural descriptions, it is puzzling that the answer cannot be read off directly from them. Perhaps this relationship is only implicit in the description, making it necessary to generate an image from the structural description and derive the answer from that.

The present volume, originally a special issue of the journal Cognition, is useful in so far as it delineates the state of the art in a few branches of vision. It makes it clear that we have advanced mainly by discovering and making precise an increasing number of problems, and that theorizing is more rigorous than ever before. But although the secret of how the brain subserves vision has for some time appeared to be just around the corner, it still remains elusive. How, for instance, does my brain calculate that my typewriter lies to the left of my gin bottle?

Stuart Sutherland is Director of the Centre for Research on Perception and Cognition, University of Sussex, Brighton BNI 9QG, UK.

\section{Morphological eye}

\section{Malcolm Irving}

Skeletal Muscle. Handbook of Microscopic Anatomy, Vol.2, Part 6. By Henning Schmalbruch. Springer-Verlag: 1985. Pp. 440. DM 580.

THE PREVIOUS edition of this handbook was published in 1956, a time of revolution in muscle research. The idea that contraction involved the relative sliding of two sets of filaments was new, and the first clear electron micrographs of the side-byside array of interdigitating filaments were still to be published. In the 30 years since then electron microscopes seem to have been focused on every structural feature in muscle, so that we now have a fairly complete picture of its ultrastructure, at least down to a resolution of about $10 \mathrm{~nm}$. Parallel progress with other techniques has in many cases helped to reveal the functional significance of the structural specializations.

This new edition is therefore long overdue and, in attempting to review progress since the last one, Schmalbruch was confronted with an embarrassment of riches. $\mathrm{He}$ has reduced his subject to manageable proportions by concentrating on one approach to the study of muscle, which he describes as "muscle seen through the eyes of a morphologist". This allows him to treat a wide range of biologically interesting phenomena from a unified viewpoint. The morphological aspects of neuromuscular transmission, contractile proteins and the mechanism of force generation, internal membranes and the regulation of contraction, metabolism and fibre types, development and regeneration are all dealt with in some depth. A wide range of other features, for example connective tissue, vascularization and the organization of motor units, are described more briefly. The emphasis is on mammalian muscle (including a section on non-skeletal striated types, but excluding cardiac muscle), though results from studies on other animals are discussed where they serve as useful experimental models. Pathological states are mentioned in passing but not treated systematically.

Schmalbruch has painstakingly organized and cross-referenced his material. which is also beautifully illustrated by over 200 micrographs and backed up by about 2,000 references. He does not hesitate to pepper the text with quantitative detail where it is available (thus we learn that a $10 \mathrm{~cm}$ cell from a human biceps contains 3,000 nuclei), or to resort to descriptions of "amorphous densities" or "ill-defined periodic structures" where it is not. Technical issues, such as possible problems in the use of a particular fixative, are discussed where they might affect the interpretation of the literature. The rather empirical "morphologist's eye" approach adopted for most of the book often leaves the reader to draw his or her own conclusions from the data; sometimes, though, as in the section on myogenesis, Schmalbruch allows himself to discuss alternative hypotheses, giving freer rein to the morphologist's brain.

This book is more than an atlas of muscle structure, but less than a complete account of the structural basis of muscle function, and it will probably be of more use to the research worker than to the undergraduate. It should remain a useful reference volume for a long period - if not quite until the publication of the next edition, which at the present rate should appear sometime after the year 2010 .

Malcolm Irving is in the Department of Biophysics, King's College London (KQC), $26-$ 29 Drury Lane, London WC2B $5 R L$, UK. 